\title{
Facial Plexiform Neurofibroma as an Intraparotid Nodule: A Rare Case Presentation
}

\author{
Shagufta Qadri ${ }^{1}$, Divya Rabindranath ${ }^{2}$, Shakeba Quadri ${ }^{3}$, Rana K Sherwani ${ }^{4}$
}

\begin{abstract}
Neurofibromas of salivary gland are very rare and account for only $0.4 \%$ of all salivary gland neoplasms. Plexiform type of neurofibromas are predominantly seen in a scenario of neurofibromatosis-1 (NF-1) association, but solitary plexiform neurofibroma has also been occasionally reported. We present a report of this variant in a 17-year-old male who is presented with a slow-growing painless swelling in the right preauricular region for 8-month duration. Microscopic examination revealed multiple cell types including Schwann cells, perineurial cells, axons, lymphocytes, mast cells, and endoneurial fibroblasts. Immunostaining with $\mathrm{S} 100$ confirmed the neural origin.

Keywords: Facial nerve, Neurofibromatosis, Parotid gland.

Otorhinolaryngology Clinics: An International Journal (2020): 10.5005/jp-journals-10003-1344
\end{abstract}

\section{INTRODUCTION}

Tumors of neural origin are rare in salivary glands. Plexiform neurofibromas are benign neoplasms of Schwann cells of the peripheral nerve sheath, which although rarely involve the salivary glands but are often present in the parotid gland. They are more commonly seen in the orbit, neck, back, and inguinal region. Plexiform type is an infrequent growth presenting as either solitary or multiple lesions frequently in the background of neurofibromatosis-1 (NF-1), also known as Von Recklinhausen's disease, which is an autosomal dominant genetically inherited disease caused by a germline mutation in the NF-1 tumor suppressor gene located at 17q11.2 encoding for neurofibromin. It can cause a range of clinical manifestations from pain, disfigurement, neurological deficits, to even life-threatening complications. Plexiform neurofibromas are difficult to be excised completely because of their diffuse tortuous growth involving multiple nerve fascicles and infiltrating nature with extension into the adjacent surrounding tissues.

The report here describes the extremely rare occurrence of a solitary plexiform neurofibroma localized to the parotid gland in the absence of neurofibromatosis syndrome, in a 17-year-old male. Though majority occur as a component of NF-1, they are not considered pathognomonic of neurofibromatosis. Reports of isolated plexiform neurofibroma without NF-1 are present in various medical literature. ${ }^{1-3}$ Patients with NF-1 and plexiform neurofibromas have a higher mortality rate in comparison to patients with or without asymptomatic plexiform neurofibromas. ${ }^{4}$

\section{Case Description}

A 17-year-old male was presented to the maxillofacial surgery outpatient department with swelling in the right side of the face that had been present for 8 months. The swelling had gradually increased in size. The patient complained of mild facial weakness; however, symptoms of paresthesia and numbness were not present. On extraoral examination, a right-sided preauricular swelling measuring $5 \times 4 \mathrm{~cm}$ was seen. The swelling was freely mobile, nontender, firm in consistency, and the overlying skin was normal. On systemic examination, there was no other swelling, café au lait
${ }^{1-4}$ Department of Pathology, Jawahar Lal Nehru Medical College, Aligarh, Uttar Pradesh, India

Corresponding Author: Shagufta Qadri, Department of Pathology, Jawahar Lal Nehru Medical College, Aligarh, Uttar Pradesh, India, Phone: +91 9897532879, e-mail: qadridrshagufta@gmail.com

How to cite this article: Qadri S, Rabindranath D, Quadri S, et al. Facial Plexiform Neurofibroma as an Intraparotid Nodule: A Rare Case Presentation. Int J Otorhinolaryngol Clin 2020;12(1):8-10.

Source of support: Nil

Conflict of interest: None

spots, or axillary freckle on the body. Family history of such lesion or NF was absent.

Computed tomography (CT) scan showed a large round hypoattenuated mass lesion with well-defined margins arising within the right parotid gland, without involvement of bone (Fig. 1A). Fine-needle aspiration cytology was inconclusive. Superficial parotidectomy was done. Peroperatively, a bulky lobulated tumor mass was seen compressing the main trunk of facial nerve; however,
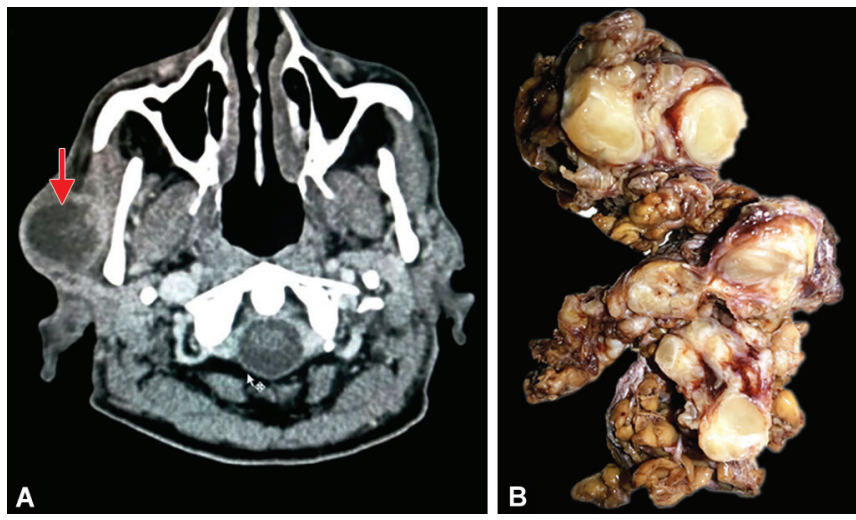

Figs $1 \mathrm{~A}$ and $\mathrm{B}$ : (A) Computed tomography scan showing ill-defined hypoattenuated mass arising in the parotid gland; (B) Cut section showing lobulated mass, with multiple white, smooth glistening nodules 

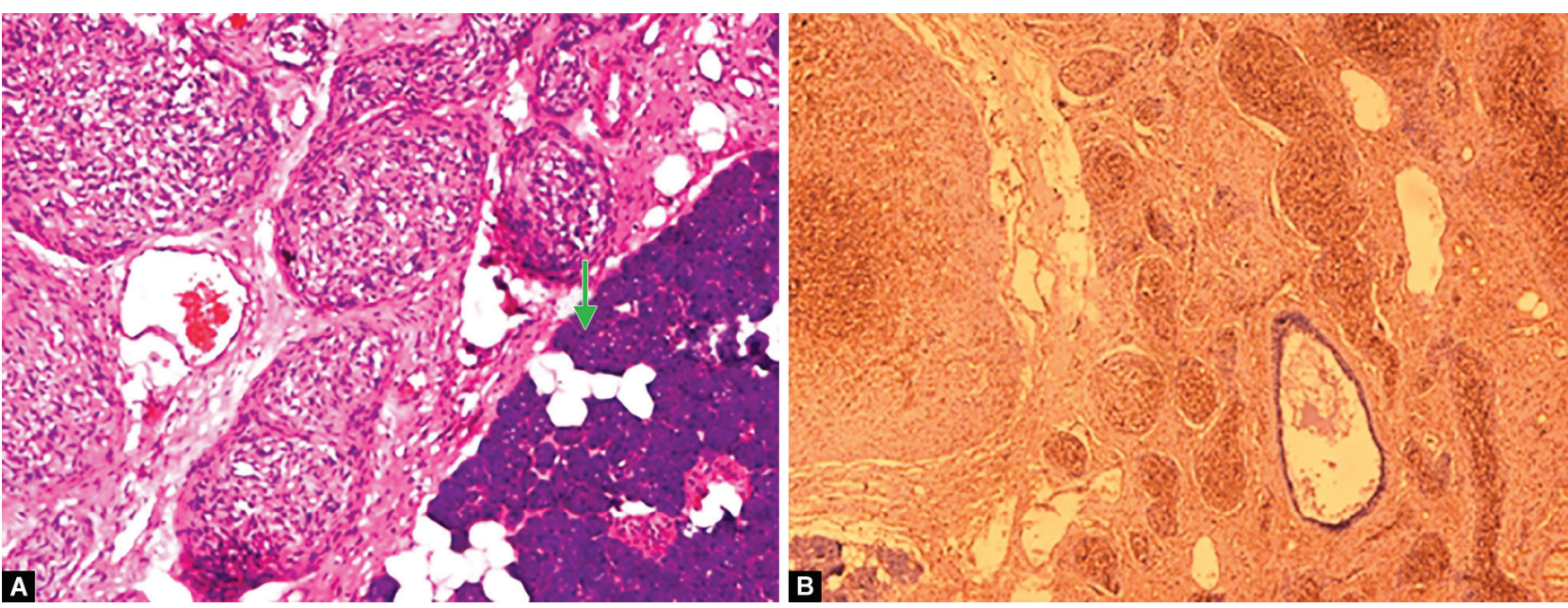

Figs $2 A$ and B: (A) Section showing tumor consisting of spindle-shaped cells in a collagenous stroma with normal serous salivary gland (H\&E, $\times 100)$; (B) Section showing $\mathrm{S} 100$ positivity of nerve fibers $(\mathrm{H} \& \mathrm{E}, \times 40)$

taking all precautions the lesion was removed atraumatically. Gross examination showed a gray-white, soft to firm mass measuring $5 \times 4.5 \times 3 \mathrm{~cm}$ with irregular, prominently enlarged tortuous nerve within the tumor nodules. The cut section was lobulated with white, smooth glistening nodules (Fig. 1B). On histopathological examination at low magnification, numerous nodules of thickened nerve bundles were seen accompanied with normal serous salivary gland (Fig. 2A). On higher magnification, tortuous nodules of expanded nerve bundles were seen in anastomosing each other. Tumor cells showed wavy serpentine nuclei with pink fibrillary cytoplasm in a myxo-collagenous background, infiltrated by few mast cells. S100 immunostain showed nuclear as well as cytoplasmic positivity (Fig. 2B) confirming its neural origin. A diagnosis of plexiform neurofibroma was thus confirmed upon histopathological examination including the immunohistochemistry.

After surgery, the patient recovered well and was discharged in good conditions without any loss of facial nerve function. The patient was followed up for 1 year with no recurrence.

\section{Discussion}

Neurofibromas are benign tumors that originate from the nerve tissue. They may be solitary or multiple, sporadic, or associated with neurofibromatosis I or II syndromes. According to the World Health Organization, neurofibromas are classified into two broad categories: dermal and plexiform neurofibromas, both being included as grade I tumors. Dermal neurofibroma involve a single peripheral nerve, while plexiform type involves multiple nerve bundles.

Plexiform neurofibromas are typically slow-growing, painless, and locally infiltrative tumors. ${ }^{5}$ Plexiform neurofibromas represent $14 \%$ of all benign mesenchymal tumors and $10 \%$ of nonepithelial salivary gland tumors. ${ }^{6}$ The common sites of occurrence are orbit, face, neck, retroperitoneum, inguinal region, chest, or abdomen. They are often found in young children, sometimes congenital. The clinical signs and symptoms are varied depending on the site of the tumor and the subsequent involvement of surrounding structures, ranging from pain to neurological deficits. Plexiform neurofibroma of parotid gland is extremely rare. Majority of tumors of parotid gland are benign, and the most common tumor of parotid gland is pleomorphic adenoma. ${ }^{7}$
Plexiform neurofibromas are one of the most common and debilitating complications of neurofibromatosis 1 syndrome, so a diligent search for any two of seven clinical features should be noted in the patient, namely, six or more café au lait spots, lisch nodules, and axillary freckles; two cutaneous neurofibromas; or one plexiform neurofibroma, sphenoid wing dysplasia, optic nerve gliomas, and any first-degree relative affected by NF-1. The NF-1 gene mapped to chr 17q11.2 encodes for neurofibromin, which has growth regulatory properties and is normally expressed in neurons, Schwann cells, glial cells, and melanocytes. The non-myelinated Schwann cells ensheath small-diameter PNS axons with their cytoplasmic processes and constitute the Remak bundle, that can undergo biallelic inactivation of NF-1 gene, thus contributing to the neoplastic proliferation in case of neurofibromas. ${ }^{8}$

Computed tomography imaging may show multilobulated masses that may appear as a "bag of worms." A characteristic "branching" hypoattenuated mass on $\mathrm{CT}$, with branching tubular masses extending into the adjacent tissue, could virtually be diagnostic. Grossly, plexiform neurofibromas are not encapsulated and typically appear as an intrafascicular diffuse tortuous nodular growth along multiple branches of more proximal nerves, resulting in a "string of onion" appearance. Histopathologically, there is diffuse cylindrical enlargement of multiple fascicles of the nerve and its contiguous branches. They are usually uniformly hypocellular with a myxoid matrix or fine fibrillary collagenous matrix containing Schwann cells, nerve fibers, mast cells, and perineurial, endoneurial fibroblasts. The cells are spindle-shaped with scanty but extremely thin elongated eosinophilic fibrillary cytoplasm with wavy or comma-shaped nuclei. It has been hypothesized that the neoplastic nonmyelinated Schwann cells secrete many chemoattractants that recruit various cell types. The role of mast cells in neurofibroma formation is to secrete mitogens necessary for favorable tumor microenvironment. Plexiform schwannoma can be a close mimicker sometimes, because of the presence of plexiform or network like anastomosing focal areas; however, the presence of a well-encapsulated lesion grossly along with certain microscopic features like verocay bodies, dimorphic growth pattern of Antoni $A$ and $B$ areas, and hyaline thickening of vessels is unique to schwannoma, which helps it in differentiating these two entities. Also plexiform schwannomas 
arise more frequently in superficial dermis or subcutaneous tissue. Malignant transformation in schwannoma is an exceptionally very rare event, in contrast to plexiform neurofibromas that have $2-5 \%$ tendency for malignant transformation. ${ }^{9}$ Accelerated growth can be seen during early childhood, puberty, and pregnancy without malignant transformation. Alarming signs including rapid increase in size, change in the consistency, increasing intensity of pain, or neurological symptoms should warrant immediate medical attention and alert the clinician toward progression of malignancy. A thorough histopathological examination with immunohistochemistry for S100 suggesting neural origin is essential for a confirmatory diagnosis of plexiform neurofibroma. Surgical excision is the treatment of choice. The recurrence is seen in about $20 \%$ of the patients with a plexiform neurofibroma after complete resection and it increases to $44 \%$ with subtotal resection. ${ }^{10}$

\section{Conclusion}

- Plexiform neurofibroma, although rare, should be considered in differential diagnosis of isolated salivary gland swellings, although it is of neurogenic rather than salivary gland origin.

- Suspicion for NF-1 should be worked out, as plexiform neurofibromas associated with NF-1 have a high mortality rate.

- Long-term clinical follow-up is mandated because of risk of recurrence and probable occurrence of malignant transformation.

\section{References}

1. Rajendran R. Plexiform neurofibroma of the gingival: report of a rare case. J oral and maxillofacial pathol 2006;10:28-30. DOI: 10.4103/0973-029X.37798.

2. Kamra HT, Dantkale SS, Birla K, et al. Plexiform neurofibroma in the floor of the mouth but without neurofibromatosis-1: a rare case report. Ecancer 2013;7:313. DOI: 10.3332/ecancer.2013.313.

3. Marocchio LS, Pereira MC, Soares CT. Oral neurofibroma not associated with neurofibromatosis type I: Case Report. J Oral Sci 2006;48(3):157-160. DOI: 10.2334/josnusd.48.157.

4. Prada CE, Rangwala FA, Martin LJ, et al. Pediatric plexiform neurofibromas: impact on morbidity and mortality in neurofibromatosis type I. J Pediatr 2012;160:461-467. DOI: 10.1016/ j.jpeds.2011.08.051.

5. Keel SB, Rosenberg AE. Soft tissue pathology of the head and neck. In: Pilch BZ. ed. Head and Neck Surgical Pathology. Philadelphia: Lippincott Williams and Wilkins; 2001. pp. 417-418.

6. Mc Daniel RK. Benign mesenchymal neoplasms in surgical pathology of the salivary glands. ed.: Ellis GL, Auclair PL, Gnepp DR, Philadelphia: WB Saunders; 1991. pp. 496-497.

7. Speight PM, Barrett AW. Salivary gland tumours. Oral Dis 2002;8: 229-240. DOI: 10.1034/j.1601-0825.2002.02870.x.

8. Zheng H, Chang L, Patel N, et al. Induction of Abnormal Proliferation by Nonmyelinating Schwann Cells Triggers Neurofibroma Formation. Cancer Cell 2008;13(2):117-128. DOI: 10.1016/j.ccr.2008.01.002.

9. Hope DG, Mulvihill J. Malignancy in neurofibromatosis. Adv Neurol 1981;29:33-56.

10. Aribandi M, Wood WE, Elston DM, et al. CT features of plexiform neurofibroma of the submandibular gland. Am J Neuroradiol 2006;27:126-128. 